\title{
Toxicity evaluation of biodegradable chitosan nanoparticles using a zebrafish embryo model
}

\author{
This article was published in the following Dove Press journal: \\ International Journal of Nanomedicine \\ I3 December 201 I \\ Number of times this article has been viewed
}

\section{Yu-Lan $\mathrm{Hu}^{\prime}$ \\ Wang $\mathrm{Qi}^{1}$ \\ Feng $\mathrm{Han}^{2}$ \\ Jian-Zhong Shao ${ }^{3}$ \\ Jian-Qing Gao'}

'Institute of Pharmaceutics, College of Pharmaceutical Sciences, ${ }^{2}$ Institute of Pharmacology, Toxicology and Biochemical Pharmaceutics, ${ }^{3}$ College of Life Sciences, Key Laboratory for Cell and Gene Engineering of Zhejiang Province, Zhejiang University, Hangzhou, Zhejiang, People's Republic of China
Correspondence: Jian-Qing Gao Institute of Pharmaceutics, College of Pharmaceutical Sciences, Zhejiang University, Hangzhou 310058,

People's Republic of China

Tel +8657188208437

Fax +86 57| 88208437

Email gaojianqing@zju.edu.cn
Background: Although there are a number of reports regarding the toxicity evaluation of inorganic nanoparticles, knowledge on biodegradable nanomaterials, which have always been considered safe, is still limited. For example, the toxicity of chitosan nanoparticles, one of the most widely used drug/gene delivery vehicles, is largely unknown. In the present study, the zebrafish model was used for a safety evaluation of this nanocarrier.

Methods: Chitosan nanoparticles with two particle sizes were prepared by ionic cross-linking of chitosan with sodium tripolyphosphate. Chitosan nanoparticles of different concentrations were incubated with zebrafish embryos, and $\mathrm{ZnO}$ nanoparticles were used as the positive control.

Results: Embryo exposure to chitosan nanoparticles and $\mathrm{ZnO}$ nanoparticles resulted in a decreased hatching rate and increased mortality, which was concentration-dependent. Chitosan nanoparticles at a size of $200 \mathrm{~nm}$ caused malformations, including a bent spine, pericardial edema, and an opaque yolk in zebrafish embryos. Furthermore, embryos exposed to chitosan nanoparticles showed an increased rate of cell death, high expression of reactive oxygen species, as well as overexpression of heat shock protein 70 , indicating that chitosan nanoparticles can cause physiological stress in zebrafish. The results also suggest that the toxicity of biodegradable nanocarriers such as chitosan nanoparticles must be addressed, especially considering the in vivo distribution of these nanoscaled particles.

Conclusion: Our results add new insights into the potential toxicity of nanoparticles produced by biodegradable materials, and may help us to understand better the nanotoxicity of drug delivery carriers.

Keywords: chitosan, nanoparticles, zebrafish embryo, nanotoxicology

\section{Introduction}

With the rapid development of nanotechnology, there is a growing interest in the application of nanomaterials in various fields, such as photonics, catalysis, magnetics, and biotechnology including cosmetics, pharmaceutics, and medicines. ${ }^{1-4}$ There are several reports regarding the toxicity evaluation of inorganic nanomaterials. ${ }^{5,6}$ However, little is known about the potential toxicity of nanoparticles used in targeted drug delivery and gene therapies in terms of human health. The risk these nanoparticles may pose to human health must be addressed, because it has been shown that nanoparticles can be administered to the human body by several routes, including inhalation, ingestion, dermal penetration, and injection, followed by distribution of these nanoparticles to various tissues through the systemic circulation. ${ }^{7,8}$

Until now, various site-specific drug targeting strategies using nanoparticle drug carrier systems have been developed, and nanoparticle-based drug targeting delivery 
systems have been introduced in the treatment of various diseases. ${ }^{9,10}$ Chitosan [poly (1,4- $\beta$-d-glucopyranosamine)], a natural polysaccharide prepared by $\mathrm{N}$-deacetylation of chitin, has great potential as a biomaterial for the construction of nanosized drug carriers and gene transfer vectors. These carriers have been the focus of considerable attention due to their many advantages, including good stability and simple preparation. ${ }^{9}$ In addition, chitosan nanoparticles can facilitate drug delivery across cellular barriers and transiently cause opening of the tight junctions between epithelial cells. ${ }^{11}$ Therefore, the possibility exists that chitosan nanoparticles may translocate from the gastrointestinal tract, nasal cavity, or alveolar sacs into the systemic circulation, thereby causing toxicity to the human body. Several studies addressing the cytotoxicity of chitosan nanoparticles have been reported. ${ }^{12-15}$ It was shown that chitosan nanoparticles with a particle size of $40 \mathrm{~nm}$ elicited dose-dependent inhibitory effects on the proliferation of a tumor cell line, and it appeared that chitosan nanoparticles had much higher cytotoxicity than chitosan. ${ }^{15}$ Other reports also indicated that chitosan nanoparticles (110-390 $\mathrm{nm}$ ) had cytotoxicity profiles against A549 cells comparable with those of the corresponding dissolved chitosan materials, with cell viability generally not affected by sample concentrations lower than $740 \mu \mathrm{g} /$ mL. ${ }^{12,14}$ Further, researchers have recently used live human cells to examine the uptake and cytotoxicity of chitosan nanoparticles, and found that they could be internalized by the cells, reducing cell viability and proliferation and compromising cell membrane integrity. ${ }^{13}$ However, the toxicity profile of chitosan nanoparticles in the body has seldom been investigated. Due to their special physicochemical properties, including a large surface area that can enhance their biological effect, nanoparticles may cause toxicity after entering the body. ${ }^{16}$ Therefore, evaluation of the potential toxic effects of these nanoparticles in the body is required, given that specific mechanisms and pathways through which nanoparticles may exert their toxic effects remain largely unknown. Until now, there has been little information on the impact of chitosan nanoparticles on human health. Further, systems for evaluation of nanoparticle toxicity are not well established. $\mathrm{ZnO}$ nanoparticles were previously evaluated for toxicity using a zebrafish model,, 6 and it was found that these nanoparticles caused toxicity to zebrafish embryos and larvae, reducing the hatching rate and causing pericardial edema. In the present study, we used $\mathrm{ZnO}$ nanoparticles as a positive control to evaluate the toxicity of chitosan nanoparticles.
Recently, the zebrafish has become a prominent vertebrate model for assessing the toxicity of drugs and chemicals. ${ }^{17-20}$ The zebrafish offers a variety of advantages as a desirable model for in vivo high-throughput drug screening. It is low cost and transparent, and its small size allows it to be assayed in 96-well plates. The embryos can develop promptly into larvae within 5 days of fertilization. As a vertebrate, the genomes of zebrafish and humans are closely related. ${ }^{19,21}$ The zebrafish is also an ideal model for the screening of different nanomaterials and for obtaining nanotoxicity information at the whole animal level, which can then be extrapolated to humans and other vertebrates. ${ }^{6,22}$ As shown in Figure 1, nanotoxicity could be evaluated in a zebrafish model, and these toxicities, including a lower hatching rate, mortality, and malformations could be observed.

In this study, we prepared chitosan nanoparticles based on ionic gelation of chitosan with tripolyphosphate anions and used the zebrafish embryo as a rapid, medium throughput, cost-effective whole animal model to assess the toxicity of the biodegradable drug/gene carrier, ie, chitosan nanoparticles. Our results demonstrate that chitosan nanoparticles of different particle sizes could cause concentration-dependent toxicity with regard to hatching, growth, and development of zebrafish embryos. Furthermore, it was demonstrated that embryos exposed to chitosan nanoparticles exhibited an increased rate of cell death and enhanced expression of reactive oxygen species, as well as overexpression of heat shock protein 70 (HSP70), indicating that chitosan nanoparticles cause damage to zebrafish embryos. We hope that our discoveries will provide more insight into the potential toxicity of nanoparticles produced by biodegradable materials and will help in understanding better the nanotoxicity of drug delivery carriers.

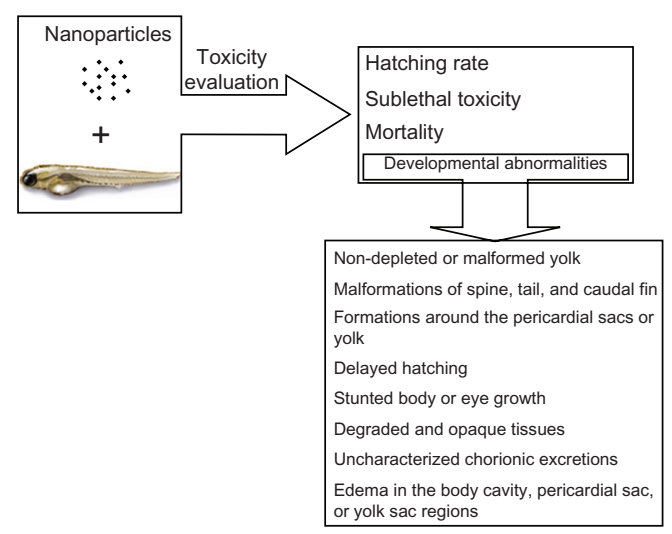

Figure I Toxicity evaluation of nanoparticles using zebrafish model. ${ }^{18}$ Reprinted from Adv Drug Deliv Rev, vol 6I, issue 6, Fako and Furgeson, Zebrafish as a correlative and predictive model for assessing biomaterial nanotoxicity, p. 478-486, Copyright (2009), with permission from Elsevier. 


\section{Materials and methods \\ Experimental fish}

Zebrafish (Danio rerio), 1 year of age and $2-3 \mathrm{~cm}$ in length, were kept in recirculating water at $26^{\circ} \mathrm{C}$, and fed with commercial pellets at a daily ration of $0.7 \%$ of their body weight. All fish were held in the laboratory for at least two weeks prior to the experiments for acclimatization and evaluation of overall fish health. The authors certify that all experiments with live animals were performed in compliance with the relevant laws, and the institutional committees approved our experiments.

\section{Materials}

Chitosan ( $85 \%$ deacetylation, molecular weight $\left.10^{5} \mathrm{Da}\right)$ was purchased from Yuhuan Oceanic Biochemistry (Taizhou, China). $\mathrm{ZnO}$ ( $>98 \%$ pure as determined by the manufacturer) with a particle size of $30 \mathrm{~nm}$ was purchased for Hangzhou Wan Jing Biotechnology Co Ltd (Hangzhou, China). Bovine serum albumin and a $2^{\prime}, 7^{\prime}$-dichlorodihydrofluorescein diacetate (H2DCF-DA) fluorescent probe was purchased from Sigma-Aldrich Inc (St Louis, MO). Nitrocellulose membrane (Hybond) was obtained from Amersham Pharmacia, Piscataway, NJ. Rabbit antirat HSP70 antibody and horseradish peroxidase-labeled antirabbit IgG were purchased from Wuhan Boshide Company (Wuhan, China). Acridine orange was purchased from Beyotime (Shanghai, China).

\section{Preparation and characterization of nanoparticles}

Chitosan solution $2.5 \mathrm{mg} / \mathrm{mL}$ was prepared by dissolving the polymer in $1 \%(\mathrm{v} / \mathrm{v})$ acetic acid aqueous solution for 0.5 hours under magnetic stirring. The $\mathrm{pH}$ of the solution was adjusted to $5.0-6.0$ using $1 \mathrm{~mol} / \mathrm{L} \mathrm{NaOH}$. The chitosan solution was then stirred for 0.5 hours at room temperature. Finally, sodium tripolyphosphate (TPP), the counter ion, was dissolved in pure water to prepare a $1 \mathrm{mg} / \mathrm{mL}$ solution, and added to the chitosan solution under mild magnetic stirring to form chitosan nanoparticles. The nanoparticle solution was centrifuged at $18,000 \mathrm{rpm}$ and $4{ }^{\circ} \mathrm{C}$ for 30 minutes, after which the nanoparticles at the bottom were collected, extensively washed with water to remove the TPP and the acetic acid, and finally lyophilized. By adjusting the mass ratio of TPP and chitosan, chitosan nanoparticles of different sizes could be prepared.

$\mathrm{ZnO}$ nanoparticles $100 \mathrm{mg} / \mathrm{L}$ were prepared in zebrafish culture medium (consisting of $64.75 \mathrm{mg} / \mathrm{L} \mathrm{NaHCO}_{3}$, $5.75 \mathrm{mg} / \mathrm{L} \mathrm{KCl}, 123.25 \mathrm{mg} / \mathrm{L}, \mathrm{MgSO}_{4} \cdot 7 \mathrm{H}_{2} \mathrm{O}$, and $294 \mathrm{mg} / \mathrm{L}$
$\mathrm{CaCl}_{2} \cdot 2 \mathrm{H}_{2} \mathrm{O}$ ), which was prepared according to the International Organization for Standardization, Geneva, Switzerland, standard 7346-3:1996 $6^{23}$ as the stock solution.

Morphological examination of the nanoparticles was performed by transmission electron microscopy. The nanoparticles were stained with $2 \%(\mathrm{w} / \mathrm{v})$ phosphotungstic acid solution for 10 seconds, immobilized on copper grids with formvar, and dried overnight before microscopy. The particle sizes were determined by laser diffraction spectrometry (Malvern Zetasizer 3000HS; Malvern, Worcestershire, UK).

\section{Exposure of zebrafish embryos to nanoparticles}

Zebrafish embryos were collected $4-5$ hours post-fertilization from the zebrafish aquarium at the Department of Pharmacy, Zhejiang University, and staged according to standard procedures. ${ }^{23}$ For the toxicity studies, 20 healthy embryos were transferred to the wells of a 24-well plate along with $2 \mathrm{~mL}$ of zebrafish culture medium. Different concentrations of chitosan nanoparticles $(10,20$, and $40 \mu \mathrm{g} / \mathrm{mL})$, with particle sizes of $200 \mathrm{~nm}$ or $340 \mathrm{~nm}$ and dissolved using zebrafish culture medium were added to the wells and incubated for 72 hours at $28.5^{\circ} \mathrm{C} . \mathrm{ZnO}$ nanoparticles $10 \mathrm{mg} / \mathrm{L}$ was used as a positive control. Tests were performed in triplicate. The hatching rate, mortality, and malformations of zebrafish embryos at 96 hours post-fertilization were observed under stereomicroscopes (Jiangnan, China) to evaluate the toxicity of the chitosan nanoparticles. The mortality rate was expressed as the number of dead embryos after 96 hours post-fertilization as compared with the control group. The hatching rate was expressed as the number of embryos that had hatched by 96 hours post-fertilization, as compared with the control group. Morphological anomalies, including chorion with attached debris, delayed development, lack of spontaneous movement at 24 hours post-fertilization, pericardial edema, yolk sac edema, bent trunk, tail malformation, and an uninflated swim bladder were observed under stereomicroscopes.

\section{Acridine orange staining}

To investigate the role of apoptosis in the toxicity of chitosan nanoparticles, acridine orange staining of nanoparticle-treated or -untreated embryos was performed. ${ }^{24}$ After exposure to $200 \mathrm{~nm}$ chitosan nanoparticles for 96 hours post-fertilization, the embryos were taken out, washed using phosphate-buffered saline, transferred to Eppendorf tubes, and stained with $100 \mu \mathrm{L}$ acridine orange $5 \mu \mathrm{g} / \mathrm{mL}$ for 
20 minutes at room temperature. The embryos were then rinsed quickly in phosphate-buffered saline before being examined under an inverse microscope.

\section{Analysis of intracellular reactive oxygen species in zebrafish embryos}

To determine the mechanisms underlying chitosan nanoparticle-mediated toxicity, intracellular reactive oxygen species were examined in zebrafish embryos because these have been implicated in the oxidative stress associated with nanotoxicity. ${ }^{25}$ Intracellular reactive oxygen species were measured by flow cytometry using a cell-permanent H2DCF-DA fluorescent probe as a reactive oxygen species indicator. The acetates of colorless H2DCF-DA are cleaved by intracellular esterase and converted to fluorescent (dichloro) fluorescein in the presence of reactive oxygen species, which can be detected by flow cytometry. For reactive oxygen species analyses, the larvae were collected at 96 hours post-fertilization and rinsed quickly using culture medium. Homogenation was conducted to prepare a single cell suspension. The cell suspension obtained was incubated with $1.25 \mathrm{mg} / \mathrm{L} \mathrm{H} 2 \mathrm{DCF}-\mathrm{DA}$ for 20 minutes in the dark and at $28.5^{\circ} \mathrm{C}$ in a fish room. The cells were then washed and resuspended in phosphate-buffered saline. The cells were subsequently analyzed by flow cytometry using a FACScan flow cytometer (Becton Dickinson, San Jose, CA). At least 10,000 events were collected. The analysis was performed using Cell Quest Software (Becton Dickinson).

\section{Western blot analysis}

To investigate the developmental toxicity of chitosan nanoparticles and to compare the toxicity caused by $\mathrm{ZnO}$ nanoparticles, Western blot analysis was performed to evaluate the protein expression of HSP70 in whole zebrafish larvae. The larvae were collected and rinsed with phosphatebuffered saline, followed by extraction of protein. The same amount of protein extracts from zebrafish larvae were loaded on each lane, electrophoresed and subsequently blotted on a nitrocellulose membrane (Hybond). The membrane was then blocked in Tris-buffered saline containing $2 \%$ bovine serum albumin and $0.05 \%$ Tween- 20 at $4^{\circ} \mathrm{C}$ overnight and then incubated with rabbit antirat $\mathrm{HSP} 70$ antibody $(1: 2000)$ at $37^{\circ} \mathrm{C}$ for 2 hours, followed by staining with horseradish peroxidaselabeled antirabbit IgG. After washing with Tris-buffered saline for three times, the membrane was incubated with a horseradish peroxidase-conjugated secondary antibody and visualized with an enhanced chemiluminescence detection system and X-ray film.

\section{Statistical analysis}

Statistical evaluation of differences between experimental group means was done by analyses of variance and Student's $t$-tests using Excel software (v 2003; Microsoft, Inc, Redmond, WA), and the results are presented as the mean \pm standard error of the mean. A value of $P<0.05$ was considered to be statistically significant and $P<0.01$ was considered to be very significant. Data points were obtained from at least three independent experiments.

\section{Results}

\section{Characterization of nanoparticles}

Chitosan nanoparticles with two kinds of particle size were successfully prepared by ionic cross-linking of chitosan with TPP. As shown in Figure 2, the chitosan nanoparticles maintained a round shape with an almost homogeneous structure, being on average $200 \pm 6 \mathrm{~nm}$ and $340 \pm 10 \mathrm{~nm}$ in diameter.

\section{Toxicity of chitosan nanoparticles in zebrafish embryos}

To assess the toxicity of the chitosan nanoparticles, the zebrafish embryo model was used to determine whether and how chitosan nanoparticles influence embryonic development. In this study, zebrafish embryos were exposed to chitosan nanoparticles for 96 hours, and dose-dependent inhibition of embryo hatching was induced by chitosan nanoparticles of different particle sizes, as shown in Figure 3. Compared with the control group, a significant decrease $(P<0.05)$ in hatching rate was observed at $20 \mathrm{mg} / \mathrm{L}$ and $40 \mathrm{mg} / \mathrm{L}$ concentrations for the $340 \mathrm{~nm}$ chitosan nanoparticles (Figure 3A). For the $200 \mathrm{~nm}$ chitosan nanoparticles, as compared with the control group, a significant or very significant decrease in hatching rate was observed for $30 \mathrm{mg} / \mathrm{L}$ and $40 \mathrm{mg} / \mathrm{L}$ chitosan nanoparticles $(200 \mathrm{~nm})$, respectively (Figure 3B). Therefore, at high concentrations, the chitosan nanoparticles had toxic effects on the zebrafish embryos.

As shown in Figure 3C and D, a toxic effect of the chitosan nanoparticles was also detected by observation of mortality among zebrafish embryos. Mortality was both dose-dependent and time-dependent (data not shown). As compared with the control group, both $30 \mathrm{mg} / \mathrm{L}$ chitosan nanoparticles $(200 \mathrm{~nm})$ and $40 \mathrm{mg} / \mathrm{L}$ chitosan nanoparticles $(340 \mathrm{~nm})$ has significant effects on mortality in zebrafish embryos. All the embryos died after exposure to the $40 \mathrm{mg} / \mathrm{L}$ chitosan nanoparticles $(200 \mathrm{~nm})$ within 96 hours of exposure. Also, surviving embryos in the $5 \mathrm{mg} / \mathrm{L}$ group $(200 \mathrm{~nm})$ showed signs of malformation, including a bent spine and an opaque yolk 
A

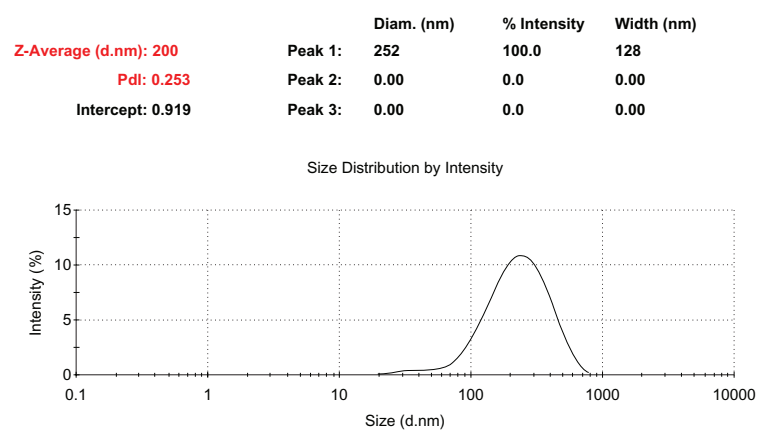

C

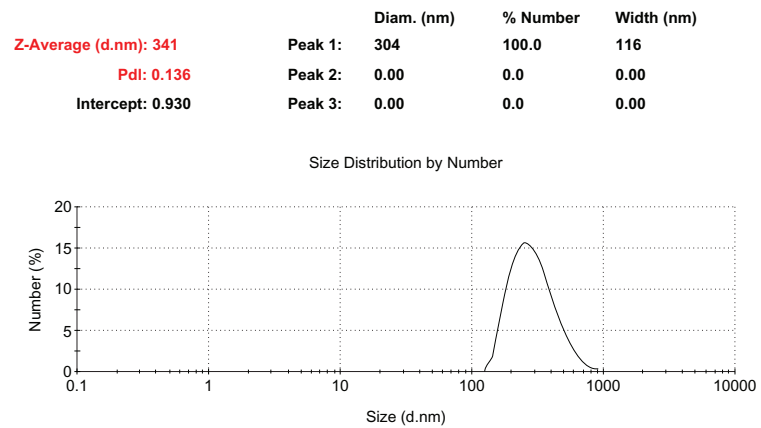

B

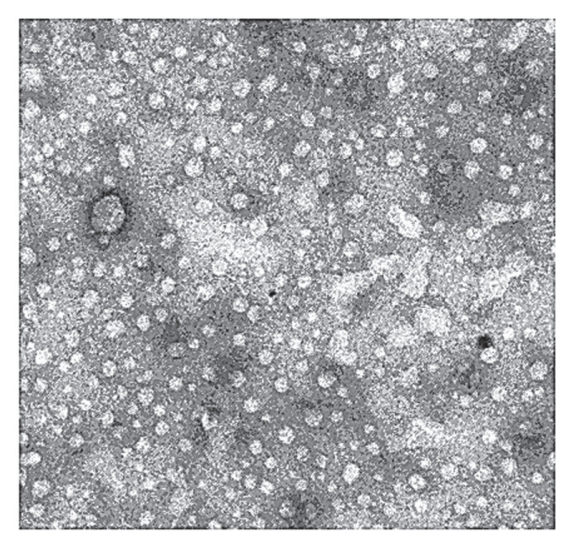

D

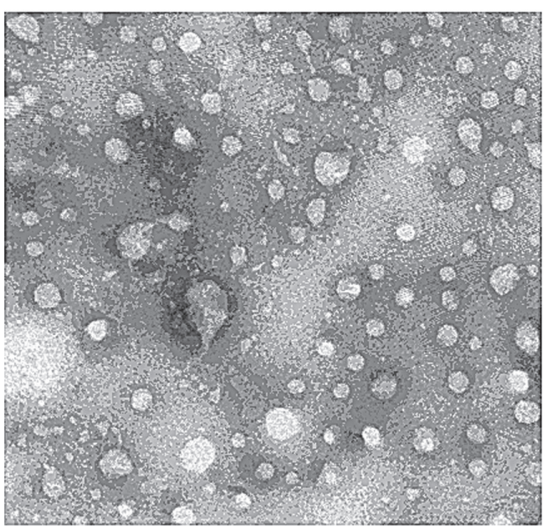

Figure 2 Characterization of $200 \mathrm{~nm}$ and $340 \mathrm{~nm}$ chitosan nanoparticles. (A) Particle size of chitosan nanoparticles measured by laser diffraction spectrometry, (B) transmission electron micrograph of $200 \mathrm{~nm}$ chitosan nanoparticles, (C) particle size of chitosan nanoparticles measured by laser diffraction spectrometry, (D) transmission electron micrograph of $340 \mathrm{~nm}$ chitosan nanoparticles.

(Figure 3E and F) as compared with controls (Figure 3G). The rate of malformation was increased with increasing chitosan nanoparticle concentration. However, embryos treated with $340 \mathrm{~nm}$ chitosan nanoparticles showed no significant malformations when compared with the $200 \mathrm{~nm}$ chitosan nanoparticle-treated group. Therefore, $200 \mathrm{~nm}$ chitosan nanoparticles had greater toxic effects than $340 \mathrm{~nm}$ chitosan nanoparticles on zebrafish embryos.

\section{Chitosan nanoparticle-induced cellular apoptosis}

To determine whether in vivo chitosan nanoparticle exposure induced cellular apoptosis in the zebrafish embryo, acridine orange staining was performed to detect cellular death in live embryos. Acridine orange is a nucleic acid-selective metachromatic dye, which emits green fluorescence upon intercalation with DNA and RNA, and is widely used for detecting sites of apoptosis in zebrafish. Acridine orange can permeate apoptotic cells and binds to DNA, whereas normal cells are nonpermeable to acridine orange. Cellular apoptosis was determined using acridine orange staining of larvae exposed to chitosan nanoparticles at 96 hours post-fertilization. In the control group, low natural fluorescence was observed, while a bright green spot could be observed on the larvae exposed to both $20 \mathrm{mg} / \mathrm{L}$ and $30 \mathrm{mg} / \mathrm{L}$ chitosan nanoparticles (Figure 4A), indicating that these particles could cause cellular apoptosis.

\section{Analysis of intracellular reactive oxygen species in zebrafish embryos}

To determine the mechanism underlying chitosan nanoparticle-mediated toxicity, intracellular reactive oxygen species were measured. ${ }^{25}$ In the present study, we tried to measure and compare intracellular reactive oxygen species in an embryonic cell suspension treated with chitosan nanoparticles. It was shown that the percentages of positive cells in $5 \mathrm{mg} / \mathrm{L}$ treated groups $(31.1 \% \pm 1.89 \%)$ were all significantly higher $(P<0.01)$ than in the control groups $(10.0 \% \pm 0.87 \%$, Figure 4B). Therefore, an increase in reactive oxygen species was found in embryos exposed to $5 \mathrm{mg} / \mathrm{L}$ chitosan nanoparticles, indicating that a higher level of cellular oxidative stress may be caused by treatment with chitosan nanoparticles. This increase in reactive oxygen 
A

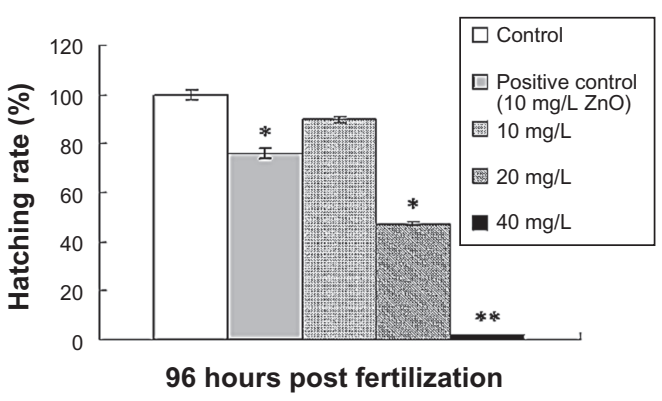

C

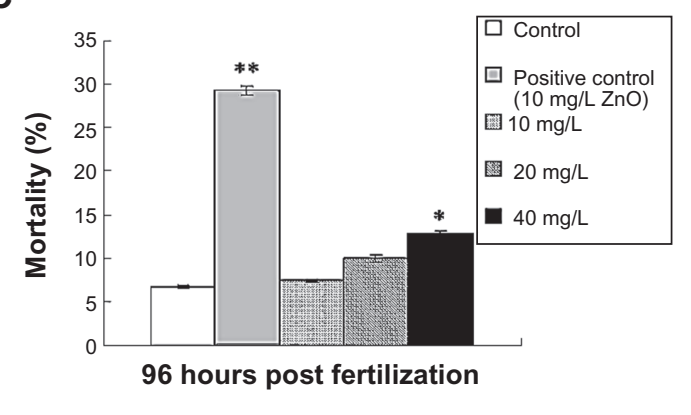

E

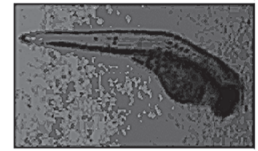

B

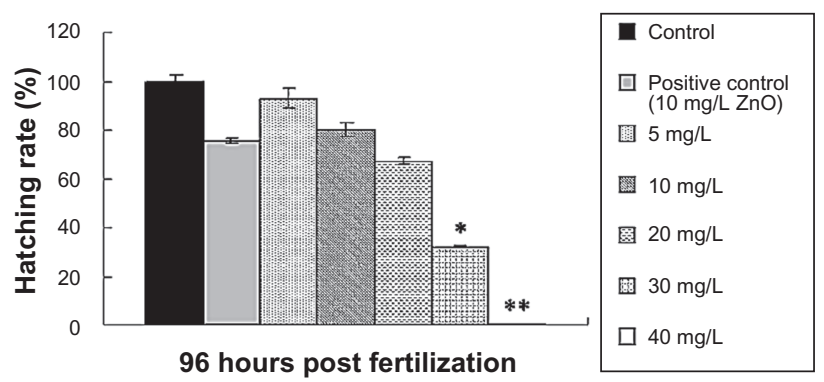

D

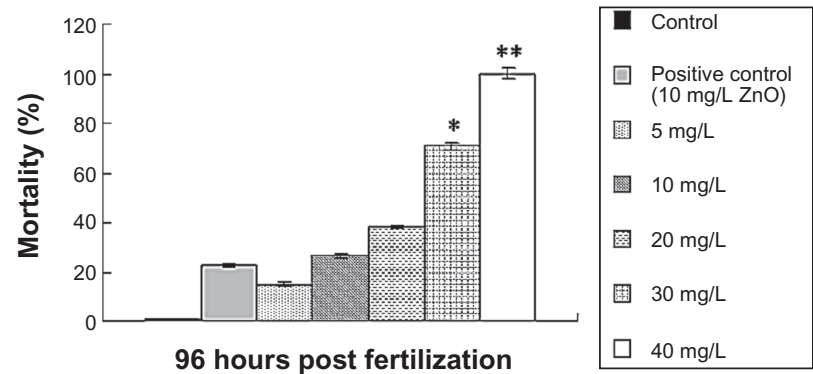

G
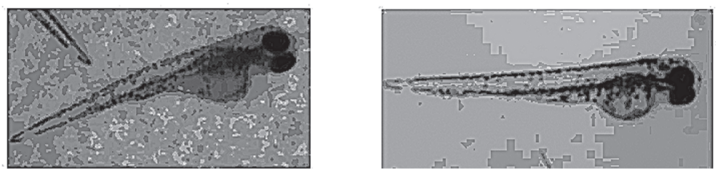

Figure 3 Toxicity of chitosan nanoparticles in zebrafish embryo. Hatching rate (\%) of zebrafish embryos exposed to (A) $340 \mathrm{~nm}$ chitosan nanoparticles or (B) $200 \mathrm{~nm}$ chitosan nanoparticles over 96 hours. Mortality of zebrafish embryos at 96 hours post-fertilization following exposure to (C) $340 \mathrm{~nm}$ or (D) $200 \mathrm{~nm}$ chitosan nanoparticles over 96 hours. Examples of malformation in zebrafish larvae exposed to $5 \mathrm{mg} / \mathrm{L} 200 \mathrm{~nm}$ chitosan nanoparticles. (E) Larva with bent trunk at 96 hours post-fertilization, (F) larva with yolk sac edema at 96 post-fertilization, and (G) control larva at 96 post-fertilization.

Note: ${ }^{*} P<0.05$ and ${ }^{* *} P<0.01$ vs water control.

species may be associated with the observed developmental toxicity of chitosan nanoparticles.

\section{Western blot analysis of HSP70 protein}

In order to characterize HSP70 expression, we examined protein expression in zebrafish larvae using Western blot analysis. HSP70 protein in control embryos is detectable. Following 96 hours of exposure to $200 \mathrm{~nm}$ chitosan nanoparticles, HSP70 protein levels increased significantly in the larvae (Figure 4C). Elevated HSP70 expression was also evident in the $\mathrm{ZnO}$ group, indicating that both $30 \mathrm{~nm} \mathrm{ZnO}$ nanoparticles and chitosan nanoparticles could be stressful stimuli for zebrafish embryos.

\section{Discussion}

Zebrafish embryos have several advantages as an animal model for the study of nanoparticle toxicity. Zebrafish have recently emerged as a model for toxicological study of various nanoparticles. For example, $30 \mathrm{~nm} \mathrm{ZnO}$ nanoparticles were found to cause dose-dependent toxicity in zebrafish embryos. When the concentrations exceeded $2 \mathrm{mg} / \mathrm{L}$, death of the zebrafish occurred and mortality increased with increasing particle concentration. Furthermore, $100 \%$ mortality was observed at a concentration of $30 \mathrm{mg} / \mathrm{L},{ }^{5}$ and $80 \mathrm{~nm}$ copper nanoparticles were found to be acutely toxic to zebrafish, with a 48 -hour LC50 concentration of $1.5 \mathrm{mg} / \mathrm{L} \cdot{ }^{26}$ It was also demonstrated that exposure to single-walled carbon nanotubes induces significantly delayed hatching in zebrafish embryos 52-72 hours post-fertilization at concentrations greater than $120 \mathrm{mg} / \mathrm{L} .{ }^{27}$ Although many studies have reported nanoparticle toxicity using the zebrafish model, these embryo toxicity studies were mostly carried out in relation to an evaluation of the toxicity of environmental nanoparticles/pollutants. ${ }^{22,27-29}$ Relatively few studies have used zebrafish to evaluate the toxicity of nanocarriers, such as biodegradable nanoparticles, which are widely used in the pharmaceutical area. Because these 
A
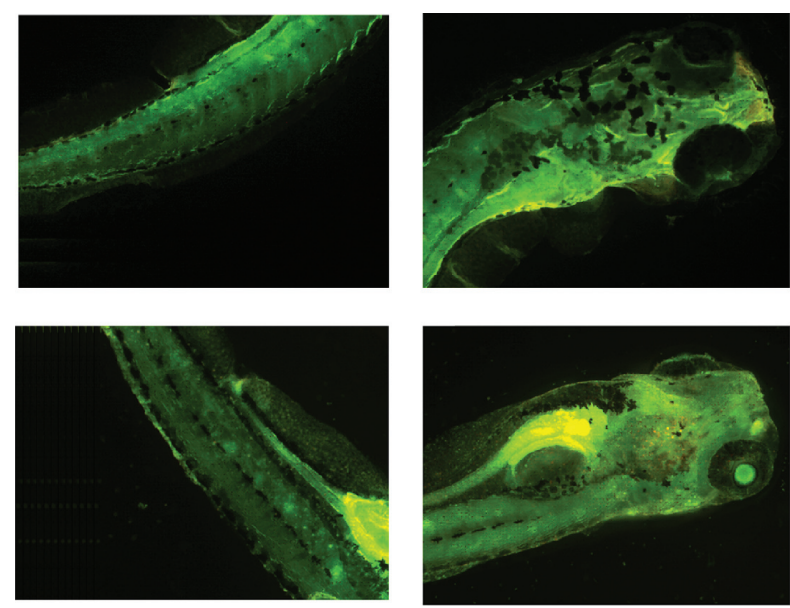

B

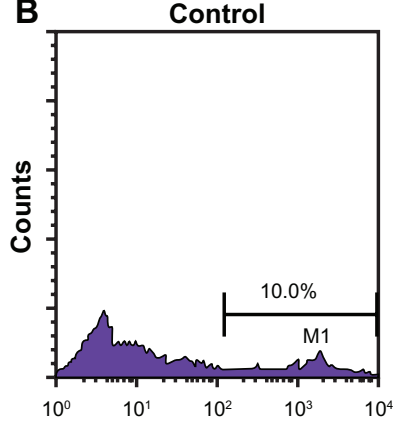

C

1

2

3

4

5

HSP70
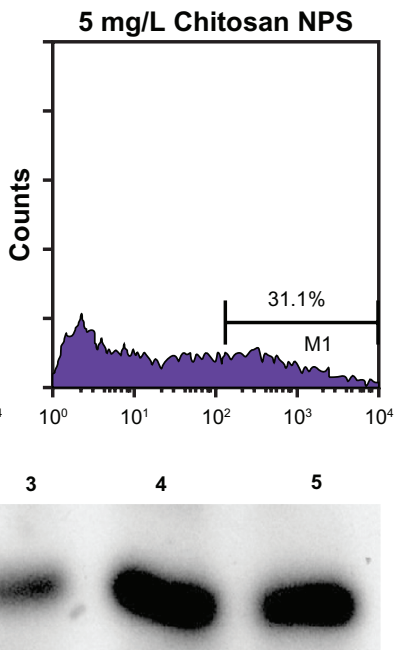

Figure $\mathbf{4}$ Investigation of the mechanism of toxicity caused by chitosan nanoparticles. (A) Cellular apoptosis was determined using acridine orange staining of chitosan nanoparticle-exposed embryos at 96 post-fertilization. Treated larvae showing bright green spots on the skin indicate the presence of apoptotic cells. Upper panel, control larvae; lower panel, larvae exposed to chitosan nanoparticles $20 \mathrm{mg} / \mathrm{L}$. (B) Flow cytometric analysis of intracellular reactive oxygen species in the control group (left panel) and $5 \mathrm{mg} / \mathrm{L}$ chitosan nanoparticle-treated group (right panel). (C) Western blot analysis of HSP70 in zebrafish embryos.

Notes: I, control group; 2, treated with $10 \mathrm{mg} / \mathrm{mL}$ chitosan nanoparticles; 3 , treated with $20 \mathrm{mg} / \mathrm{mL}$ chitosan nanoparticles; 4 , treated with $40 \mathrm{mg} / \mathrm{mL}$ chitosan nanoparticles; 5 , treated with $5 \mathrm{mg} / \mathrm{L} \mathrm{ZnO}$ nanoparticles.

nanomaterials possess unique physical and surface properties, they have inspired plans for a wide spectrum of applications, such as target-specific vehicles for in vivo sensing, diagnosis, and therapy (eg, nanomedicine, drug delivery). However, after entering the body, they may show cytotoxicity and immune toxicity because of their distribution in the body, changes in cellular affinity, and increased cellular uptake. ${ }^{30-32}$ After systemic administration, these nanoparticles are small enough to penetrate very small capillaries throughout the body, and

therefore could be a very effective approach to target certain tissues $^{33}$ and may affect the physiology of many cells in the animal body. ${ }^{34}$

Several reports have shown that nanoparticles can enter the brain and cause tissue injury. ${ }^{4,35}$ Therefore, evaluation of the toxicity of these nanocarriers is important for human health. Unfortunately, the evaluation of nanoparticle toxicity remains limited. In the present study, zebrafish embryos were explored as an effective model for toxicity evaluation of chitosan nanoparticles. No apparent toxic effects were observed for chitosan nanoparticles at low concentrations. However, death and malformation of zebrafish embryos occurred with increasing chitosan nanoparticle concentrations. Almost $100 \%$ mortality was observed at a concentration of $40 \mathrm{mg} / \mathrm{L}$ for the $200 \mathrm{~nm}$ chitosan nanoparticles. Therefore, the toxicity was dose-dependent and should be considered at high concentrations. Although there was no decrease in hatching rate or mortality at a concentration of $5 \mathrm{mg} / \mathrm{L}$ for both $200 \mathrm{~nm}$ and $300 \mathrm{~nm}$ chitosan nanoparticles, malformation was found at this concentration for $200 \mathrm{~nm}$ chitosan nanoparticles, while no malformation was detected for $300 \mathrm{~nm}$ chitosan nanoparticles. Therefore, the $200 \mathrm{~nm}$ nanoparticles showed higher toxicity than the $300 \mathrm{~nm}$ nanoparticles in the zebrafish embryo model. Meanwhile, $200 \mathrm{~nm}$ chitosan nanoparticles at a concentration of $5 \mathrm{mg} / \mathrm{L}$ were examined for production of reactive oxygen species in zebrafish embryos, and higher levels were detected, which confirms the toxic effect caused by the $5 \mathrm{mg} / \mathrm{L}$ nanoparticles.

From the description in a review paper, ${ }^{9}$ chitosan nanoparticles are a promising system for drug delivery because of their biodegradability, biocompatibility, and good stability. They have been reported to have key applications in various areas including parenteral drug delivery, oral administration of drugs, vaccine delivery, ocular drug delivery, and brain targeting drug delivery. ${ }^{9}$ The preparation of chitosan nanoparticles was mainly by the ionotropic gelation method. Using this method, the chitosan nanoparticles prepared were mostly around 200-400 nm diameter, which was normally used in the drug delivery system. For example, estradiol-loaded chitosan nanoparticles with an average size of $269.3 \pm 31.6 \mathrm{~nm}$ were prepared by Wang et al. ${ }^{36}$ Aktas et al prepared chitosan nanoparticles containing a caspase inhibitor with a particle diameter ( $Z$ average) ranging from approximately $313 \mathrm{~nm}$ to $412 \mathrm{~nm} .{ }^{37}$ Sadeghi et al ${ }^{38}$ prepared chitosan-insulin nanoparticles with a diameter of $250 \pm 20 \mathrm{~nm}$. It was also reported that the particle size of unloaded chitosan nanoparticles and doxorubicin-loaded nanoparticles was 
$259 \pm 15 \mathrm{~nm}$ and $292 \pm 42 \mathrm{~nm}$, respectively. ${ }^{39}$ Recently, we prepared Ganoderma lucidum polysaccharide-loaded chitosan nanoparticles with a mean diameter of $217 \pm 6 \mathrm{~nm} \cdot{ }^{40}$ Following on from the aforementioned literature reports, we prepared blank chitosan nanoparticles of two different sizes, ie, $200 \pm 6 \mathrm{~nm}$ and $340 \pm 10 \mathrm{~nm}$, for toxicity evaluation in zebrafish.

The mechanism underlying the toxicity of chitosan nanoparticles was also explored by evaluation of cellular apoptosis, expression of reactive oxygen species, and HSP70. As shown by acridine orange staining, apoptosis could be seen in the embryos after exposure to the nanoparticles. Increased production of intracellular reactive oxygen species was also detected, indicating chitosan nanoparticles caused oxidative stress, as a result of the observed toxicity in zebrafish embryos. We also observed higher expression of HSP70 protein in the nanoparticleexposed group, indicating stress was caused by the chitosan nanoparticles. Heat shock proteins could be induced by a variety of stimuli, and are potentially damaging to the cell. They are associated with many cellular processes including protein synthesis, folding and translocation, and assembly of larger protein complexes, all of which can be impaired by stress. ${ }^{41}$ HSP70, one of the major heat shock proteins, is usually expressed at low levels, but increases in response to environmental and physiological stressors. ${ }^{42}$ HSP70 is conserved throughout evolution and has been shown to protect cells against induction of cell death by a variety of stresses and different modes of cell death. ${ }^{42}$

Although more and more research is being done using the zebrafish model for drug discovery and drug screening, ${ }^{20,43}$ few studies have assessed the toxicity of nanocarriers using this model. Here, we first report concentration-dependent toxicity of biodegradable chitosan nanoparticles. However, in evaluation of nanoparticle toxicity, the surface area, surface charge, coating, material solubility, and exposure time are also expected to play important roles, and thus should be carefully considered in future work.

\section{Acknowledgments}

We thank Cheng Chengzhi, from Zhejiang University, China, for his technical assistance. We thank Yang Zhou from Iowa State University for polishing this article. This work was supported in part by the National Basic Research Program of China (2009CB930300), the 46th China Postdoctoral Grant (20090461389), and the Medical Scientific Research Foundation of Zhejiang Province, China (2010 KYB035).

\section{Disclosure}

The authors report no conflicts of interest in this work.

\section{References}

1. Donaldson K. Resolving the nanoparticles paradox. Nanomedicine (Lond). 2006;1(2):229-234.

2. Kagan VE, Bayir H, Shvedova AA. Nanomedicine and nanotoxicology: two sides of the same coin. Nanomedicine. 2005;1(4):313-316.

3. Linkov I, Satterstrom FK, Corey LM. Nanotoxicology and nanomedicine: making hard decisions. Nanomedicine. 2008;4(2):167-171.

4. Medina C, Santos-Martinez MJ, Radomski A, Corrigan OI, Radomski MW. Nanoparticles: pharmacological and toxicological significance. Br J Pharmacol. 2007;150(5):552-558.

5. Xiong D, Fang T, Yu L, Sima X, Zhu W. Effects of nano-scale TiO2, ZnO and their bulk counterparts on zebrafish: acute toxicity, oxidative stress and oxidative damage. Sci Total Environ. 2011;409(8):1444-1452.

6. Zhu X, Wang J, Zhang X, Chang Y, Chen Y. The impact of ZnO nanoparticle aggregates on the embryonic development of zebrafish (Danio rerio). Nanotechnology. 2009;20(19):195103.

7. Burch WM. Passage of inhaled particles into the blood circulation in humans. Circulation. 2002;106(20):e141-e142.

8. Takenaka S, Karg E, Roth C, et al. Pulmonary and systemic distribution of inhaled ultrafine silver particles in rats. Environ Health Perspect. 2001;109 Suppl 4:547-551.

9. Nagpal K, Singh SK, Mishra DN. Chitosan nanoparticles: a promising system in novel drug delivery. Chem Pharm Bull (Tokyo). 2010;58(11): 1423-1430

10. Agnihotri SA, Mallikarjuna NN, Aminabhavi TM. Recent advances on chitosan-based micro- and nanoparticles in drug delivery. $J$ Control Release. 2004;100(1):5-28.

11. Dodane V, Amin Khan M, Merwin JR. Effect of chitosan on epithelial permeability and structure. Int J Pharm. 1999;182(1):21-32.

12. Ma Z, Lim LY. Uptake of chitosan and associated insulin in Caco-2 cell monolayers: a comparison between chitosan molecules and chitosan nanoparticles. Pharm Res. 2003;20(11):1812-1819.

13. Loh JW, Yeoh G, Saunders M, Lim LY. Uptake and cytotoxicity of chitosan nanoparticles in human liver cells. Toxicol Appl Pharmacol. 2010;249(2):148-157.

14. Huang M, Khor E, Lim LY. Uptake and cytotoxicity of chitosan molecules and nanoparticles: effects of molecular weight and degree of deacetylation. Pharm Res. 2004;21(2):344-353.

15. Qi L, Xu Z, Jiang X, Li Y, Wang M. Cytotoxic activities of chitosan nanoparticles and copper-loaded nanoparticles. Bioorg Med Chem Lett. 2005;15(5):1397-1399.

16. Governa M, Valentino M, Visona I, Monaco F, Amati M. Importance of the specific surface area in the study of the biological effects of dusts of industrial use. G Ital Med Lav. 1995;17(1-6):105-109. Italian.

17. Wang S, Liu K, Wang X, He Q, Chen X. Toxic effects of celastrol on embryonic development of zebrafish (Danio rerio). Drug Chem Toxicol. 2011;34(1):61-65.

18. Fako VE, Furgeson DY. Zebrafish as a correlative and predictive model for assessing biomaterial nanotoxicity. Adv Drug Deliv Rev. 2009; 61(6):478-486.

19. Hill AJ, Teraoka H, Heideman W, Peterson RE. Zebrafish as a model vertebrate for investigating chemical toxicity. Toxicol Sci. 2005; 86(1):6-19.

20. McGrath P, Li CQ. Zebrafish: a predictive model for assessing druginduced toxicity. Drug Discov Today. 2008;13(9-10):394-401.

21. Yang L, Ho NY, Alshut R, et al. Zebrafish embryos as models for embryotoxic and teratological effects of chemicals. Reprod Toxicol. 2009;28(2):245-253

22. Bai W, Tian W, Zhang Z, et al. Effects of copper nanoparticles on the development of zebrafish embryos. J Nanosci Nanotechnol. 2010; 10(12):8670-8676 
23. International Organization for Standardization. Water qualitydetermination of the acute lethal toxicity of substances to a freshwater fish. [Brachydaniorerio Hamilton-Buchanan (Teleostei, Cyprinidae)]. Part 3: Flow-through method. Geneva, Switzerland: International Organization for Standardization; 1996.

24. Asharani PV, Wu YL, Gong ZY, Valiyaveettil S. Toxicity of silver nanoparticles in zebrafish models. Nanotechnology. 2008;19(25):255102.

25. Li N, Xia T, Nel AE. The role of oxidative stress in ambient particulate matter-induced lung diseases and its implications in the toxicity of engineered nanoparticles. Free Radic Biol Med. 2008;44(9):1689-1699.

26. Griffitt RJ, Weil R, Hyndman KA, et al. Exposure to copper nanoparticles causes gill injury and acute lethality in zebrafish (Danio rerio). Environ Sci Technol. 2007;41(23):8178-8186.

27. Cheng JP, Flahaut E, Cheng SH. Effect of carbon nanotubes on developing zebrafish (Danio rerio) embryos. Environ Toxicol Chem. 2007;26(4):708-716.

28. Fent K, Weisbrod CJ, Wirth-Heller A, Pieles U. Assessment of uptake and toxicity of fluorescent silica nanoparticles in zebrafish (Danio rerio) early life stages. Aquat Toxicol. 2010;100(2):218-228.

29. King-Heiden TC, Wiecinski PN, Mangham AN, et al. Quantum dot nanotoxicity assessment using the zebrafish embryo. Environ Sci Technol. 2009;43(5):1605-1611.

30. Hu YL, Gao JQ. Potential neurotoxicity of nanoparticles. Int J Pharm. 2010;394(1-2):115-121.

31. Maurer-Jones MA, Bantz KC, Love SA, Marquis BJ, Haynes CL. Toxicity of therapeutic nanoparticles. Nanomedicine (Lond). 2009; 4(2):219-241.

32. Xia T, Kovochich M, Liong M, Zink JI, Nel AE. Cationic polystyrene nanosphere toxicity depends on cell-specific endocytic and mitochondrial injury pathways. ACS Nano. 2008;2(1):85-96.

33. Braydich-Stolle L, Hussain S, Schlager JJ, Hofmann MC. In vitro cytotoxicity of nanoparticles in mammalian germline stem cells. Toxicol Sci. 2005;88(2):412-419.
34. Brooking J, Davis SS, Illum L. Transport of nanoparticles across the rat nasal mucosa. J Drug Target. 2001;9(4):267-279.

35. Sharma HS. Nanoneuroscience: emerging concepts on nanoneurotoxicity and nanoneuroprotection. Nanomedicine (Lond). 2007;2(6): 753-758.

36. Wang X, Chi N, Tang X. Preparation of estradiol chitosan nanoparticles for improving nasal absorption and brain targeting. Eur J Pharm Biopharm. 2008;70(3):735-740.

37. Aktas Y, Andrieux K, Alonso MJ, et al. Preparation and in vitro evaluation of chitosan nanoparticles containing a caspase inhibitor. Int J Pharm. 2005;298(2):378-383.

38. Sadeghi AM, Dorkoosh FA, Avadi MR, Saadat P, Rafiee-Tehrani M, Junginger HE. Preparation, characterization and antibacterial activities of chitosan, N-trimethyl chitosan (TMC) and N-diethylmethyl chitosan (DEMC) nanoparticles loaded with insulin using both the ionotropic gelation and polyelectrolyte complexation methods. Int J Pharm. 2008; 355(1-2):299-306.

39. Janes KA, Fresneau MP, Marazuela A, Fabra A, Alonso MJ. Chitosan nanoparticles as delivery systems for doxorubicin. J Control Release. 2001;73(2-3):255-267.

40. Li N, Hu YL, He CX, et al. Preparation, characterisation and anti-tumour activity of Ganoderma lucidum polysaccharide nanoparticles. J Pharm Pharmacol. 2010;62(1):139-144.

41. Lindquist S, Craig EA. The heat-shock proteins. Annu Rev Genet. 1988; 22:631-677.

42. Fulda S, Gorman AM, Hori O, Samali A. Cellular stress responses: cell survival and cell death. Int J Cell Biol. 2010;2010:214074.

43. Kari G, Rodeck U, Dicker AP. Zebrafish: an emerging model system for human disease and drug discovery. Clin Pharmacol Ther. 2007;82(1) $70-80$.
International Journal of Nanomedicine

\section{Publish your work in this journal}

The International Journal of Nanomedicine is an international, peerreviewed journal focusing on the application of nanotechnology in diagnostics, therapeutics, and drug delivery systems throughou the biomedical field. This journal is indexed on PubMed Central, MedLine, CAS, SciSearch $₫$, Current Contents $₫ /$ Clinical Medicine,

\section{Dovepress}

Journal Citation Reports/Science Edition, EMBase, Scopus and the Elsevier Bibliographic databases. The manuscript management system is completely online and includes a very quick and fair peer-review system, which is all easy to use. Visit http://www.dovepress.com/ testimonials.php to read real quotes from published authors. 